\title{
Thalidomide Treatment Reduces \\ Tumor Necrosis Factor $\alpha$ Production and Enhances Weight Gain in Patients with Pulmonary Tuberculosis
}

\author{
Jane M. Tramontana,* Utaiwan Utaipat, *† Anthony Molloy,* \\ Pasakorn Akarasewi, ${ }^{\neq}$Margaret Burroughs,* \\ Sanit Makonkawkeyoon, ${ }^{\dagger}$ Barbara Johnson,* \\ Jeffrey D. Klausner,\$ William Rom, $\$$ and Gilla Kaplan* \\ *Laboratory of Cellular Physiology and Immunology, The Rockefeller \\ University, 1230 York Avenue, New York, New York, U.S.A. \\ ${ }^{\dagger}$ Department Microbiology and Faculty Medicine, Chiang Mai \\ University, Chiang Mai, Thailand \\ ${ }^{\ddagger}$ Chiang Mai Anti-TB Association, Chiang Mai, Thailand \\ §Department Medicine and Pulmonary and Critical Care Medicine, \\ New York University Medical Center, 550 First Avenue, New York, \\ New York, U.S.A.
}

\begin{abstract}
Background: The monocyte-derived cytokine, tumor necrosis factor $\alpha(\mathrm{TNF} \alpha)$, is essential for host immunity, but overproduction of this cytokine may have serious pathologic consequences. Excess TNF $\alpha$ produced in pulmonary tuberculosis may cause fevers, weakness, night sweats, necrosis, and progressive weight loss. Thalidomide ( $\alpha-N$-phthalimidoglutarimide) has recently been shown to suppress TNF $\alpha$ production by human monocytes in vitro and to reduce serum TNF $\alpha$ in leprosy patients. We have therefore conducted a two-part placebo-controlled pilot study of thalidomide in patients with active tuberculosis to determine its effects on clinical response, immune reactivity, TNF $\alpha$ levels, and weight. Materials and Methods: 30 male patients with active tuberculosis, either human immunodeficiency virus type 1 positive $\left(\mathrm{HIV}-\mathrm{I}^{+}\right)$or $\mathrm{HIV}_{-} \mathrm{l}^{-}$, received thalidomide or
\end{abstract}

placebo for single or multiple 14 day cycles. Toxicity of the study drug, delayed type hypersensitivity (DTH), cytokine production, and weight gain were evaluated.

Results: Thalidomide treatment was well tolerated, without serious adverse events. The drug did not adversely affect the DTH response to purified protein derivative (PPD), total leukocyte, or differential cell counts. TNF $\alpha$ production was significantly reduced during thalidomide treatment while interferon- $\gamma$ (IFN $\gamma$ ) production was enhanced. Daily administration of thalidomide resulted in a significant enhancement of weight gain.

Conclusions: The results indicate that thalidomide is well tolerated by patients receiving anti-tuberculosis therapy. Thalidomide treatment reduces TNF $\alpha$ production both in vivo and in vitro and is associated with an accelerated weight gain during the study period.

\section{INTRODUCTION}

Tumor necrosis factor $\alpha$ (TNF $\alpha)$ is one of the important immunologic mediators generated by cells of the monocyte/macrophage lineage and

Address correspondence and reprint requests to: Gilla Kaplan, The Rockefeller University, 1230 York Avenue, New York, NY 10021, U.S.A. has been shown to have significant effects on host immunity in bacterial and parasitic infections (1). Tuberculosis is a chronic mycobacterial infection (of macrophages and monocytes) in which TNF $\alpha$ is characteristically produced. TNF $\alpha$ is present in the pleural effusions of patients infected with Mycobacterium tuberculosis, and monocytes isolated from patients with active dis- 
ease and systemic symptoms release elevated amounts of TNF $\alpha$ into the culture supernatant in response to stimuli (2-4). In vitro, mycobacterial preparations have been shown to induce TNF $\alpha$ production by human monocytes (5). TNF $\alpha$ is essential for granuloma formation $(6,7)$, which is intimately associated with resistance against mycobacteria (8).

While production of TNF $\alpha$ is essential for host immunity, overproduction of this cytokine may have serious pathologic consequences. In experimental animals, high levels of TNF $\alpha$ induce fever by direct action on the hypothalamus (1), and long-term administration of this cytokine causes marked losses of fat and muscle cells or cachexia (9). Elevated levels of TNF $\alpha$ produced in pulmonary tuberculosis may be responsible for many of the pathologic symptoms of the disease, including fevers, weakness, fatigue, night sweats, lung necrosis, and progressive weight loss (10).

The control of these pathologic effects of excess TNF $\alpha$ production has received increasing attention. In animal models of gram-negative sepsis, administration of either antibodies directed against TNF $\alpha$ or pharmaceutical agents that suppress TNF $\alpha$ production, leads to reduced fevers, less wasting, and significantly decreased mortality (11). One drug, thalidomide $(\alpha-N$-phthalimidoglutarimide), has recently been shown to suppress $\mathrm{TNF} \alpha$ production by human monocytes in vitro $(12,13)$ and to normalize elevated levels of serum TNF $\alpha$ in vivo $(12,14)$. The inhibitory activity of thalidomide is due to a selective destabilization of the TNF $\alpha$ mRNA (15); thalidomide selectively suppresses TNF $\alpha$ production in vitro without directly affecting the levels of other cytokines such as interleukin-1 (IL-1), IL-6, and granulocyte/macrophage-colony stimulating factor (GM-CSF) (13).

Since thalidomide inhibits but does not abolish TNF $\alpha$ production, thalidomide therapy might reduce the toxicities linked with overproduction of the cytokine, without interfering with its important role(s) in host immunity. Recent studies have shown that thalidomide has prompt and dramatic effects when given to leprosy patients with erythema nodosum leprosum (ENL), including the elimination of the lesions, a decrease in inflammation, and general enhancement of patient well-being. These studies suggest that thalidomide may play a therapeutic role in tuberculosis patients, where much of the pathology may be TNF $\alpha$ mediated, but where a normal immune response and granuloma formation is important in the control of the infection.
In order to determine the effect of thalidomide on TNF $\alpha$ levels and whether thalidomide treatment is associated with any untoward side effects, including drug toxicity or suppression of immune reactivity, we have conducted a twopart placebo-controlled pilot study of thalidomide in patients with active tuberculosis. The results of our study indicate that thalidomide reduces TNF $\alpha$ production by peripheral blood mononuclear cells (PBMCs) in patients receiving anti-tuberculosis therapy without any adverse effects. Furthermore, although all patients showed the normal expected response to antituberculosis therapy, only patients treated with thalidomide demonstrated significant increased weight gain during the study period.

\section{MATERIALS AND METHODS}

\section{Study Drug}

Thalidomide (CG-217) was provided in 100-mg tablets (Lot 204 and 206) by Grunenthal GMBH (Aachen, Germany). Placebo tablets were also provided by Grunenthal GMBH (Aachen, Germany).

\section{Study Design}

A total of 30 male patients with active tuberculosis, either HIV- $1^{+}$or HIV-1 ${ }^{-}$, were studied. Twenty of the patients were studied in Chiang Mai, Thailand (Group I), and 10 were studied in New York City, U.S.A. (Group II). In Thailand, patients were evaluated at the Tuberculosis Center in Chiang Mai and admitted to the Tuberculosis Sanitorium. In New York, patients were transferred from Bellevue Hospital to the Clinical Research Center (CRC) at the Rockefeller University Hospital.

All patients received $300 \mathrm{mg}$ of thalidomide or placebo in tablet form ( 3 tablets) daily at bedtime. The Thailand patients received only a single 14-day cycle of thalidomide or placebo. In New York, after the first treatment cycle with thalidomide or placebo, Patients 25-30 were allowed a 7-day washout period and then treated with a second, third, and in some a fourth cycle of thalidomide or placebo. Of the $10 \mathrm{New}$ York patients, one completed only 7 days of thalidomide during the initial cycle of treatment (Patient 21); the remaining nine completed the full initial cycle of 14 days of thalidomide or placebo. 
INCLUSION CRITERIA. Table 1 summarizes the patient characteristics. Patients were included in the study if they had acid fast organisms on sputum examination with eventual growth of $M$. tuberculosis in culture and met one or more of the following criteria: fever greater than $38^{\circ} \mathrm{C}$; weight loss greater than $5 \mathrm{~kg}$; night sweats; and evidence of pulmonary disease on chest X-ray consistent with tuberculosis. Patients were excluded from the study if there was growth of mycobacteria other than tuberculosis or if patients refused human immunodeficiency virus type 1 (HIV-1) testing. Also patients with pre-existing peripheral neuropathy were excluded, since this is a well-described side effect of thalidomide.

CONCOMITANT THERAPIES. All patients received multidrug anti-tuberculosis regimens (MDT) as shown in Table 1. Anti-retroviral therapy was administered to the New York patients who were HIV $-1^{+}$(Table 1) but was not available for the Thailand patients. The protocol was approved by the Institutional Review Board of the Rockefeller University Hospital and by the Ministry of Health in Thailand. Written consent was obtained for all patients.

\section{Evaluation of Patients}

All patients were evaluated by history and physical exam at baseline, at multiple time points during the study, and after cessation of the drug. Vital signs, including weight, were obtained twice daily. In Thailand, complete blood counts with differentials and biochemistry profiles were obtained before, on Day 7 of treatment and 5 days after the end of drug treatment. In New York, in addition to complete blood counts and biochemistry profile, laboratory evaluation included phenotyping of blood mononuclear leukocytes. Chest radiographs were obtained at baseline and after cessation of treatment with thalidomide or placebo. Delayed type hypersensitivity (DTH) was assessed by the response to intradermal injection of $0.1 \mathrm{ml}$ of the tuberculin PPD (Mantoux, Connaught Laboratories, Inc., Swiftwater, PA, U.S.A.) and quantitated after $48 \mathrm{hr}$. DTH testing was performed at baseline, on Day 7 of treatment with drug or placebo, and 5 days after discontinuation of thalidomide or placebo. The size of the response is expressed as the mean of the two greatest diameters (in $\mathrm{mm}$ ). Anergy testing was performed using the Multitest skin test reaction (Connaught). Patients 20, 23,25 , and 26 were not available for repeated skin testing. Complete caloric intake was recorded for patients in New York (Group II) only.

PBMC PHENOTYPING. PBMCs were isolated as previously described (15). For indirect immunofluorescent staining, PBMCs were stained with a saturating amount of the following monoclonal antibodies: anti-CD3 (Leu-4), anti-CD4 (Leu3a), anti-CD8 (Leu-2a), anti-CD19 (Leu-12) (Becton Dickinson, Mountain View, CA, U.S.A.), anti-CD56 (NKHI) (Coulter, Hialeah, FL, U.S.A.), and anti-CD14 (Ortho Diagnostics, Westwood, MA, U.S.A.). Cells were then analyzed with the Becton Dickinson FACScan flow cytometer.

\section{Tissue Culture}

PBMCs were isolated from blood obtained from all patients in the study and resuspended at a density of $2 \times 10^{6} / \mathrm{ml}$ in $2 \times$ culture medium (CM). CM consisted of RPMI 1640 (Gibco, Grand Island, NY, U.S.A.) supplemented with $10 \%$ pooled human $\mathrm{AB}^{+}$serum (Biocell, Carson, CA, U.S.A.), $100 \mathrm{U}$ penicillin $/ \mathrm{ml}, 100 \mu \mathrm{g}$ streptomycin $/ \mathrm{ml}$, and $2 \mathrm{mM}$ L-glutamine (Gibco). Incomplete tissue culture results were obtained for Patients 21-23 because of tissue culture contamination; not all assays were available for Patients 20 and 30.

\section{Cytokine Measurements}

IN VITRO. Bacterial preparations: The Pasteur strain 1011 of Mycobacterium bovis Bacillus Calmette-Guérin (BCG) (Ataufo de Paiva Foundation, Rio de Janeiro, Brazil), the purified protein derivative (PPD) of $M$. tuberculosis for use in vitro (Statenseruminstitut, Copenhagen, Denmark), synthetic muramyl dipeptide (MDP) (Sigma, St. Louis, MO, U.S.A.), and gram-negative lipopolysaccharide isolated from Salmonella typhimurium (LPS) (List Biological Laboratories, Campbell, CA, U.S.A.) were obtained commercially.

For induction of cytokines, $0.1-\mathrm{ml}$ aliquots of cell suspensions were added to each well of 96well flat bottom tissue culture plates (Costar, Cambridge, MA, U.S.A.) containing $0.1-\mathrm{ml}$ aliquots of RPMI 1640 alone, or bacterial preparations suspended in RPMI 1640, to give final concentrations of $10 \mu \mathrm{g}$ PPD, $10 \mu \mathrm{g} \mathrm{BCG}, 0.5 \mu \mathrm{g}$ MDP, or 10 ng LPS per ml.

Plates were incubated for $16 \mathrm{hr}$ at $37^{\circ} \mathrm{C}$, in $5 \% \mathrm{CO}_{2}$. Then enzyme-linked immunosorbent assays (ELISA) (Endogen, Boston, MA, U.S.A.), were performed according to the manufacturer's 
TABLE 1. Patient characteristics, clinical status, and therapies

\begin{tabular}{|c|c|c|c|c|c|c|}
\hline $\begin{array}{l}\text { Patient } \\
\text { Number }\end{array}$ & $\begin{array}{l}\text { Study } \\
\text { Drug }^{a}\end{array}$ & $\begin{array}{l}\text { HIV-1 } \\
\text { Status }\end{array}$ & $\begin{array}{c}\text { Age } \\
\text { (years) }\end{array}$ & $\begin{array}{c}\text { Baseline } \\
\text { Weight } \\
\text { (kg) }\end{array}$ & $\begin{array}{l}\text { No. Lung Fields } \\
\text { Involved }^{b}\end{array}$ & Medication $^{c}$ \\
\hline \multicolumn{7}{|l|}{ Thailand } \\
\hline 1 & $t$ & + & 43 & 48.0 & 6 & Rifater, E \\
\hline 2 & $\mathrm{t}$ & + & 51 & 49.0 & 4 & Rifater, E \\
\hline 3 & $\mathrm{t}$ & - & 32 & 43.0 & 4 & Rifater, E \\
\hline 4 & $\mathrm{t}$ & - & 65 & 40.5 & 4 & Rifater, E \\
\hline 5 & $\mathrm{t}$ & - & 46 & 43.3 & 4 & Rifater, E \\
\hline 6 & $\mathrm{t}$ & + & 60 & 41.3 & 4 & RPE \\
\hline 7 & $\mathrm{t}$ & - & 31 & 52.8 & 1 & RIP \\
\hline 8 & $\mathrm{t}$ & + & 38 & 48.3 & 2 & Rifater, E \\
\hline 9 & $\mathrm{t}$ & - & 25 & 56.5 & 3 & Rifater, E \\
\hline 10 & $\mathrm{t}$ & + & 25 & 49.5 & 1 & Rifater, E \\
\hline 11 & $\mathrm{t}$ & + & 49 & 44.5 & 3 & Rifater, E \\
\hline 12 & $\mathrm{t}$ & - & 38 & 50.5 & 2 & Rifater, E \\
\hline 13 & $\mathrm{p}$ & - & 25 & 40.0 & 2 & Rifater, E \\
\hline 14 & $\mathrm{p}$ & - & 53 & 50.0 & 4 & Rifater, E \\
\hline 15 & $\mathrm{t}$ & - & 55 & 52.0 & 5 & Rifater, E \\
\hline 16 & $\mathrm{p}$ & - & 64 & 49.0 & 5 & Rifater, E \\
\hline 17 & $t$ & - & 22 & 42.0 & 1 & Rifater, E \\
\hline 18 & $\mathrm{p}$ & - & 46 & 63.3 & 1 & Rifater, E \\
\hline 19 & $t$ & - & 63 & 71.0 & 3 & Rifater, E \\
\hline 20 & $\mathrm{p}$ & - & 42 & 58.0 & NA & Rifater, E \\
\hline \multicolumn{7}{|l|}{ New York } \\
\hline 21 & $\mathrm{t}$ & + & 44 & 63.3 & 1 & RIPE + Anti retroviral \\
\hline 22 & $\mathrm{t}$ & + & 37 & 71.0 & 2 & RIPE + Anti retroviral \\
\hline 23 & $\mathrm{p}$ & + & 33 & 77.7 & 1 & RIPE + Anti retroviral \\
\hline 24 & $\mathrm{p}$ & + & 45 & 76.5 & 3 & RIPE + Anti retroviral \\
\hline 25 & $\mathrm{t} ; \mathrm{t} ; \mathrm{p} ; \mathrm{t}$ & - & 32 & 53.7 & 3 & RIPE \\
\hline 26 & $\mathrm{t} ; \mathrm{t}$ & + & 35 & 66.5 & 2 & RIPE + Anti retroviral \\
\hline 27 & $\mathrm{t} ; \mathrm{t}$ & + & 30 & 63.3 & 3 & RIPE + Anti retroviral \\
\hline 28 & $\mathrm{t} ; \mathrm{t} ; \mathrm{p} ; \mathrm{t}$ & - & 38 & 74.5 & 3 & $\mathrm{RIPE}+\mathrm{CIPI}+\mathrm{ETH} I$ \\
\hline 29 & $\mathrm{p} ; \mathrm{t} ; \mathrm{t}$ & + & 42 & 83.8 & 1 & RIPE + Anti retroviral \\
\hline 30 & $\mathrm{t} ; \mathrm{p} ; \mathrm{t} ; \mathrm{p}$ & - & 46 & 89.6 & 2 & RIPE \\
\hline
\end{tabular}

NA, not available; R, Rifampin; I, Isoniazid; P, Pyrazinamide; E, Ethambutol; CIPI, Cipro for 16 days; ETHI, Ethionamide for 16 days.

${ }^{a}$ Patients received either thalidomide $(t)$, placebo $(p)$, or multiple cycles of $t$ or $p$ in the sequence shown.

${ }^{b}$ Infiltration, granulomas, or cavities present on chest X-ray: a total of six lung fields were evaluated and results given for each patient.

'Rifater, Rifampin isoniazid pyrazinamide. 
instructions on $0.1-\mathrm{ml}$ aliquots of culture supernatants, or of 10 -fold dilutions of the same supernatants, to determine TNF $\alpha$, IL- 1 , and interferon- $\gamma$ (IFN $\gamma$ ) levels. All data points were derived from triplicate cultures.

DIRECT ASSAY OF CYTOKINES IN PATIENT SERA. Samples of serum obtained from the patients at the same time points as PBMCs were assayed directly for TNF $\alpha$, IL-1, and IFN $\gamma$ by ELISA (Endogen). All assays were performed in triplicate. Serum samples were also assayed for total TNF $\alpha$ (receptor bound as well as free bioactive TNF $\alpha$ ) by a commercial EIA (Medgenix, Fleurus, Belgium) (16).

\section{Lymphocyte Transformation Tests}

Culture conditions for lymphocyte transformation tests (LTTs) were identical to those described above except that U-bottom plates were used. After 5 days of incubation, $1 \mu \mathrm{Ci}$ of tritiated thymidine $\left(\left[{ }^{3} \mathrm{H}\right]\right)$-TdR) (New England Nuclear, Boston, MA, U.S.A.) was added to U-bottom microwells. Following a 16-hr incubation, wells were harvested onto paper mats which were thoroughly washed, dried, and immersed in scintillation fluid for counting $\beta$ emission. Data is also presented as stimulation index (SI) calculated as: (the cpm in the presence of stimulus)/ (cpm in the CM alone).

\section{Quantitative Reverse Transcriptase- Polymerase Chain Reaction Analysis of Cytokine mRNA Levels}

Freshly isolated PBMCs obtained from all group II patients (New York) were used. RNA isolated from PBMCs obtained from Group I patients (Thailand) was partially degraded and not used for analyses. Reverse transcriptase-polymerase chain reaction (RT-PCR) for cytokine mRNA was carried out as described in detail elsewhere (17). Briefly, RNA was extracted from 4-6 $\times 10^{6}$ PBMC immediately after isolation using RNAzol B (Cinna/Biotecx, Houston, TX, U.S.A.) according to the manufacturer's instructions. Absorption spectroscopy was used to measure purity and concentration of RNA with a $A_{260 / 280}$ ratio of 2.0 indicating highly purified RNA. RNA was reverse transcribed into cDNA which was then amplified using the Gene-Amp RNA-PCR kit (Perkin Elmer Cetus, Norwalk, CT, U.S.A.) with 30 cycles of $95^{\circ} \mathrm{C}$ for 1 min (denaturation) and $60^{\circ} \mathrm{C}$ for $1 \mathrm{~min}$ (primer annealing and extension). To normalize for the amount of input
RNA, RT-PCR was performed on the constitutively expressed gene encoding glyceraldehyde phosphate dehydrogenase (GA3PD). PCR products were electrophoresed and transferred to nylon membranes (Zetabind, Cuno, Inc., Meriden, CT, U.S.A.). After prehybridization, membranes were hybridized with $5 \times 10^{5} \mathrm{cpm} / \mathrm{ml}$ of ${ }^{32} \mathrm{P}$ labeled oligonucleotide probes for $8-12 \mathrm{hr}$ at $40^{\circ} \mathrm{C}$. After washing, membranes were exposed to X-ray film (Hyperfilm-MP, Amersham Corp., Arlington Heights, IL) at room temperature with an intensifier screen. Quantitative analysis by comparison with known standards, and primer and probe sequences have been detailed (17).

\section{Statistical Analysis}

Data obtained were evaluated by the Wilcoxon signed rank test (two-sided test), or by the Student's paired sample $t$ test. $p$ values of 0.05 or less were considered significant.

\section{RESULTS}

\section{Drug Tolerance and Adverse Effects}

At the inception of the study, 10 male patients (Table 1) were enrolled in Group I (Thailand). The patients had been recently diagnosed with active tuberculosis and all began anti-tuberculosis treatment with MDT simultaneously or within a few days of treatment with thalidomide. All patients received one 14-day cycle of thalidomide, as described in Materials and Methods. The thalidomide treatment was well tolerated. No peripheral neuropathy, excessive sedation, or other adverse events were observed. The only side effects noted were morning drowsiness, dry mouth, and constipation. In addition, all patients responded well to concomitant anti-tuberculosis therapy and showed clinical improvement.

Since there were no adverse effects, approval was granted for enrollment of an additional 10 patients in the study (in Thailand). These 10 additional male patients (Table 1 ) were randomized to receive either thalidomide or placebo. All patients completed the 14-day cycle of thalidomide or placebo treatment. Administration of either thalidomide or placebo did not result in any clinical or radiographic deterioration in any of the patients. Again, clinical improvement was noted after beginning anti-tuberculosis therapy. One patient (Patient 6, Table 1) developed a macular rash which resolved with discontinua- 
tion of isoniazid and hence was not associated with thalidomide treatment.

This phase of the study established that a single 14-day cycle of thalidomide treatment was well tolerated and did not induce untoward side effects. We then received (FDA and IRB) approval to extend the studies for multiple cycles of thalidomide treatment. Therefore, an additional 10 male patients, ages 30 to 46, (Group II) were enrolled in the study in New York (Table 1). All had been diagnosed with active tuberculosis, and had received anti-tuberculosis therapy for $1-4$ weeks prior to their entry into the study. During the study, 4 patients (Patients 21-24, Table 1) received a single cycle of thalidomide or placebo; the remaining 6 patients (Patients 25-30, Table 1) received multiple cycles of thalidomide and/or placebo (Table 1). All patients in Group II (New York) responded well to thalidomide treatment; there were no adverse responses in either the thalidomide- or placebo-treated patients, except for sedation, dry mouth, and constipation, as previously noted.

\section{Effect of Thalidomide Treatment on Delayed Type Hypersensitivity}

Delayed type hypersensitivity (DTH) was assessed in each patient by the skin test response to soluble antigen. The response to PPD was quantitated before, during, and after therapy to determine the effect of thalidomide on cell mediated immunity. At Day 0 (baseline) before the first cycle of thalidomide or placebo treatment, 21 patients were $\mathrm{PPD}^{+}$while 6 patients were $\mathrm{PPD}^{-}$ (nonresponsive) (Table 2). Treatment with thalidomide did not alter the DTH response to PPD in patients who were already $\mathrm{PPD}^{+}$(Table 2 ). However, in the five patients who were nonresponsive to PPD at the beginning of the treatment cycle, thalidomide administration was associated with a significantly enhanced DTH reaction. This responsiveness returned to baseline after discontinuation of thalidomide in three of the patients. Both HIV-1 ${ }^{-}$and HIV- ${ }^{+}$showed similar DTH patterns in response to thalidomide treatment.

Reactivity to the Connaught panel of antigens was also tested. Responses to Old Tuberculin were similar to those observed with PPD (not shown). No patient became anergic to this or any other antigen during the course of the study.
TABLE 2. Delayed type hypersensitivity response to PPD during first cycle of study ${ }^{a}$

\begin{tabular}{|c|c|c|c|}
\hline \multirow{2}{*}{$\begin{array}{l}\text { Patient } \\
\text { Number }\end{array}$} & \multicolumn{3}{|c|}{$\begin{array}{c}\text { Skin Test Response to PPD } \\
(\mathrm{mm})^{b}\end{array}$} \\
\hline & Pre & Mid & Post \\
\hline \multicolumn{4}{|l|}{$\mathrm{PPD}^{+}$} \\
\hline 1 & 26.5 & 20.5 & 24.0 \\
\hline 3 & 14.0 & 25.0 & 18.5 \\
\hline 4 & 19.0 & 16.0 & 20.5 \\
\hline 5 & 16.5 & 18.0 & 24.5 \\
\hline 6 & 14.0 & 12.0 & 16.5 \\
\hline 7 & 23.5 & 65.0 & $\mathrm{ND}^{c}$ \\
\hline 9 & 18.5 & 14.5 & 21.5 \\
\hline 11 & 14.5 & 18.0 & 13.0 \\
\hline 12 & 19.5 & 26.0 & 17.0 \\
\hline $13^{d}$ & 15.0 & 21.0 & 26.0 \\
\hline $14^{d}$ & 26.5 & 22.0 & 31.0 \\
\hline 15 & 16.0 & 18.0 & 10.0 \\
\hline $16^{d}$ & 26.0 & 22.5 & 23.0 \\
\hline $18^{d}$ & 20.0 & 20.5 & 17.0 \\
\hline 19 & 15.5 & 18.0 & 16.0 \\
\hline $20^{d}$ & ND & ND & ND \\
\hline 21 & 11.0 & 15.5 & 12.0 \\
\hline 22 & 22.0 & 19.0 & 17.0 \\
\hline $23^{d}$ & ND & ND & ND \\
\hline 25 & ND & ND & ND \\
\hline 26 & 19.0 & ND & ND \\
\hline 27 & 13.0 & 14.5 & 18.0 \\
\hline 28 & 13.0 & 12.5 & 17.5 \\
\hline $29^{d}$ & 18.0 & 14.0 & 10.0 \\
\hline \multicolumn{4}{|l|}{$\mathrm{PPD}^{-}$} \\
\hline 2 & 0 & 9.5 & 0 \\
\hline 8 & 0 & 19.5 & 0 \\
\hline 10 & 0 & 12.5 & 0 \\
\hline 17 & 0 & 9.0 & 11.5 \\
\hline $24^{d}$ & 0 & 0 & 0 \\
\hline 30 & 0 & 19.0 & ND \\
\hline
\end{tabular}

${ }^{a}$ Patients in Thailand and New York were skin tested $3 \times$ during the first cycle of study.

${ }^{b}$ Mean diameter of the indurated area at $48 \mathrm{hr}$ after $5 \mathrm{u}$ PPD administration. Diameter $>10 \mathrm{~mm}$ is termed PPD ${ }^{+}$. ND, not done.

${ }^{d}$ Patients received placebo during the first cycle of study. 
TABLE 3. White blood cell counts and differentials

\begin{tabular}{|c|c|c|c|c|c|}
\hline & & \multicolumn{4}{|c|}{ Cell Count per $\mathrm{mm}^{3}$ of Blood (mean $\left.\pm S D\right)$} \\
\hline & & Total WBCs & Lymphocyte & Monocyte & Eosinophil \\
\hline \multicolumn{6}{|c|}{ Thalidomide } \\
\hline \multirow[t]{3}{*}{$\mathrm{HIV}^{+}$} & pre & $6765 \pm 2286$ & $1700 \pm 927$ & $359 \pm 276$ & $318 \pm 247$ \\
\hline & mid & $6403 \pm 2489$ & $1438 \pm 669$ & $320 \pm 237$ & $463 \pm 562$ \\
\hline & post & $6331 \pm 1564$ & $1784 \pm 1165$ & $427 \pm 273$ & $703 \pm 562$ \\
\hline \multirow[t]{3}{*}{$\mathrm{HIV}^{-}$} & pre & $8435 \pm 3519$ & $1762 \pm 876$ & $579 \pm 367$ & $513 \pm 492$ \\
\hline & mid & $7560 \pm 2402$ & $2402 \pm 1294$ & $393 \pm 301$ & $524 \pm 613$ \\
\hline & post & $6941 \pm 1735$ & $2310 \pm 801$ & $454 \pm 393$ & $445 \pm 451$ \\
\hline \multicolumn{6}{|l|}{ Placebo } \\
\hline \multirow[t]{3}{*}{$\mathrm{HIV}^{+} / 1^{-}$} & pre & $7573 \pm 3289$ & $1699 \pm 440$ & $616 \pm 392$ & $195 \pm 151$ \\
\hline & mid & $6806 \pm 3035$ & $2140 \pm 1019$ & $336 \pm 198$ & $317 \pm 321$ \\
\hline & post & $6588 \pm 4012$ & $2024 \pm 806$ & $282 \pm 170$ & $178 \pm 167$ \\
\hline
\end{tabular}

Results are means \pm one standard deviation (SD) evaluated before (pre), during (mid), and 5 days after (post) 14-day treatment cycles with $300 \mathrm{mg} /$ day of thalidomide or placebo (see Table 1). No significant changes were observed. WBC, white blood cells.

\section{Effect of Thalidomide Treatment on Blood Parameters}

Complete blood counts and differential analyses were performed on all patients receiving thalidomide or placebo. Thalidomide treatment did not cause any significant changes in total leukocyte count or differential cell count (Table 3). Fluorescence-activated cell analysis (FACS) of leukocyte subsets was also carried out during the study in New York (Group II patients). No significant effect on subset distribution was observed either in thalidomide- or placebo-treated patients, regardless of whether patients were $\mathrm{HIV}-\mathrm{I}^{-}$or HIV $-\mathrm{I}^{+}$(not shown). The percent of leukocyte subsets was similar in both HIV-1- ${ }^{-}$or $\mathrm{HIV}-1^{+}$ patients receiving either placebo or thalidomide treatment. There was, however, a slight increase in $\mathrm{CD}^{+} \mathrm{T}$ cells in $\mathrm{HIV}-1^{+}$patients compared with HIV-1- ${ }^{-}$patients.

\section{Lymphocyte Function in Vitro}

PBMCs were isolated from all patients prior to, on Day 7, and after thalidomide or placebo administration, and the proliferation in vitro in response to PPD and BCG was determined. Thalidomide did not significantly affect the ability of the cells to incorporate $\left[{ }^{3} \mathrm{H}\right]-\mathrm{TdR}$ in response to the antigens tested (Table 4) although some fluctuations in the stimulation index were observed. $\mathrm{HIV}-\mathrm{I}^{+}$patients were still responsive to PPD in vitro. This responsiveness was not significantly altered by treatment with thalidomide (Table 4).

\section{Effect of Thalidomide Treatment on Cytokine Production}

IFN $\gamma$ PRODUCTION. To determine whether thalidomide treatment reduced the ability of the host to produce protective cytokines in response to infection, IFN $\gamma$ production was evaluated both in vivo in the blood of thalidomide and placebo treated individuals and in vitro, in PBMCs.

In vivo. Patients treated with thalidomide had significantly enhanced serum IFN $\gamma$ levels after 7 days of the first cycle of thalidomide treatment (Fig. 1). The serum IFN $\gamma$ levels returned to baseline upon discontinuation of drug. In contrast, there was no increase in serum IFN $\gamma$ levels in patients treated with placebo (Fig. 1).

In vitro. When PBMCs isolated from patients were stimulated with PPD, IFN $\gamma$ was released into the culture medium (Fig. 1). Similar results 
TABLE 4. Proliferative response of PBMC to PPD and BCG

\begin{tabular}{|c|c|c|c|}
\hline & \multicolumn{3}{|c|}{ Lymphocyte Proliferation-counts per minute (stimulation index) } \\
\hline & \multicolumn{2}{|c|}{ Thalidomide } & \multirow{2}{*}{ 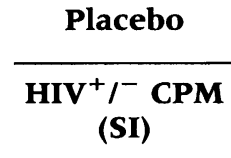 } \\
\hline & $\begin{array}{c}\text { HIV }^{+} \text {CPM } \\
\text { (SI) }\end{array}$ & $\begin{array}{c}\text { HIV }^{-} \mathbf{C P M} \\
\text { (SI) }\end{array}$ & \\
\hline \multicolumn{4}{|c|}{ Controls: } \\
\hline pre & $704 \pm 337$ & $1239 \pm 1028$ & $1037 \pm 524$ \\
\hline mid & $2264 \pm 4694$ & $2620 \pm 2949$ & $1158 \pm 664$ \\
\hline post & $1487 \pm 1694$ & $1140 \pm 664$ & $1632 \pm 1376$ \\
\hline \multicolumn{4}{|l|}{ PPD: } \\
\hline pre & $\begin{array}{c}38253 \pm 38987 \\
(60 \pm 64)\end{array}$ & $\begin{array}{c}57926 \pm 41427 \\
(64 \pm 46)\end{array}$ & $\begin{array}{c}51878 \pm 33764 \\
(63 \pm 50)\end{array}$ \\
\hline $\operatorname{mid}$ & $\begin{array}{c}49341 \pm 46031 \\
(52 \pm 56)\end{array}$ & $\begin{array}{c}44717 \pm 23963 \\
(34 \pm 31)\end{array}$ & $\begin{array}{c}60007 \pm 28475 \\
(80 \pm 75)\end{array}$ \\
\hline post & $\begin{array}{c}44077 \pm 43822 \\
(56 \pm 61)\end{array}$ & $\begin{array}{c}53078 \pm 31411 \\
(59 \pm 44)\end{array}$ & $\begin{array}{c}52682 \pm 43678 \\
(63 \pm 52)\end{array}$ \\
\hline \multicolumn{4}{|l|}{ BCG: } \\
\hline pre & $\begin{array}{c}32879 \pm 36725 \\
(42 \pm 37)\end{array}$ & $\begin{array}{c}22096 \pm 28567 \\
(28 \pm 36)\end{array}$ & $\begin{array}{c}26777 \pm 18977 \\
(32 \pm 26)\end{array}$ \\
\hline $\operatorname{mid}$ & $\begin{array}{c}44767 \pm 54076 \\
(42 \pm 58)\end{array}$ & $\begin{array}{c}23788 \pm 17519 \\
(23 \pm 32)\end{array}$ & $\begin{array}{c}34200 \pm 29931 \\
(42 \pm 53)\end{array}$ \\
\hline post & $\begin{array}{c}40172 \pm 42663 \\
(43 \pm 50)\end{array}$ & $\begin{array}{c}23259 \pm 17060 \\
(26 \pm 19)\end{array}$ & $\begin{array}{c}27891 \pm 32902 \\
(33 \pm 36)\end{array}$ \\
\hline
\end{tabular}

Results are means \pm one standard deviation $(\mathrm{SD})$ of counts per minute (stimulation indexes) of proliferative responses to antigen stimulation of PBMC tested before (pre), during (mid), and 5 days after (post) treatment with thalidomide or placebo (see Table 1). No significant changes were observed.

were observed when PBMCs from patients were stimulated with BCG (Table 5), but not with LPS or MDP (not shown). Thalidomide treatment in vivo enhanced the release in vitro of IFN $\gamma$ by PBMCs in response to mycobacterial antigens regardless of the patient's HIV status. The heightened response to both BCG and PPD was transient, and IFN $\gamma$ levels returned to baseline levels after discontinuation of thalidomide treatment (Fig. 1 and Table 5). Thalidomide treatment did not appreciably alter levels of IFN $\gamma$ mRNA expressed in freshly isolated PBMC (not shown). Placebo treatment had little effect on PPD- and BCG-stimulated release of IFN $\gamma$ by PBMCs (Fig. 1 and Table 5).

TNF $\alpha$ PRODUCTION. To determine whether treat- ment with thalidomide caused changes in production of inflammatory cytokines, PBMCs were stimulated with a variety of agonists in vitro. In one series of experiments, PBMCs isolated from all patient bloods during the first treatment cycle were treated with LPS in vitro, and TNF $\alpha$ released into the culture supernatant was quantitated. At the midpoint of thalidomide treatment ( 7 days), there was a reduction in $\mathrm{TNF} \alpha$ levels released by LPS stimulated PBMCs when compared with the prestudy levels (Fig. 2). The inhibitory effect of thalidomide treatment on TNF $\alpha$ production was observed in $\mathrm{HIV}-\mathrm{I}^{+}$as well as HIV $-1^{-}$patients. Thalidomide treatment also reduced the amount of TNF $\alpha$ produced in vitro in response to BCG (Table 5) and PPD (not shown). The thalidomide-induced reduction of TNF $\alpha$ re- 

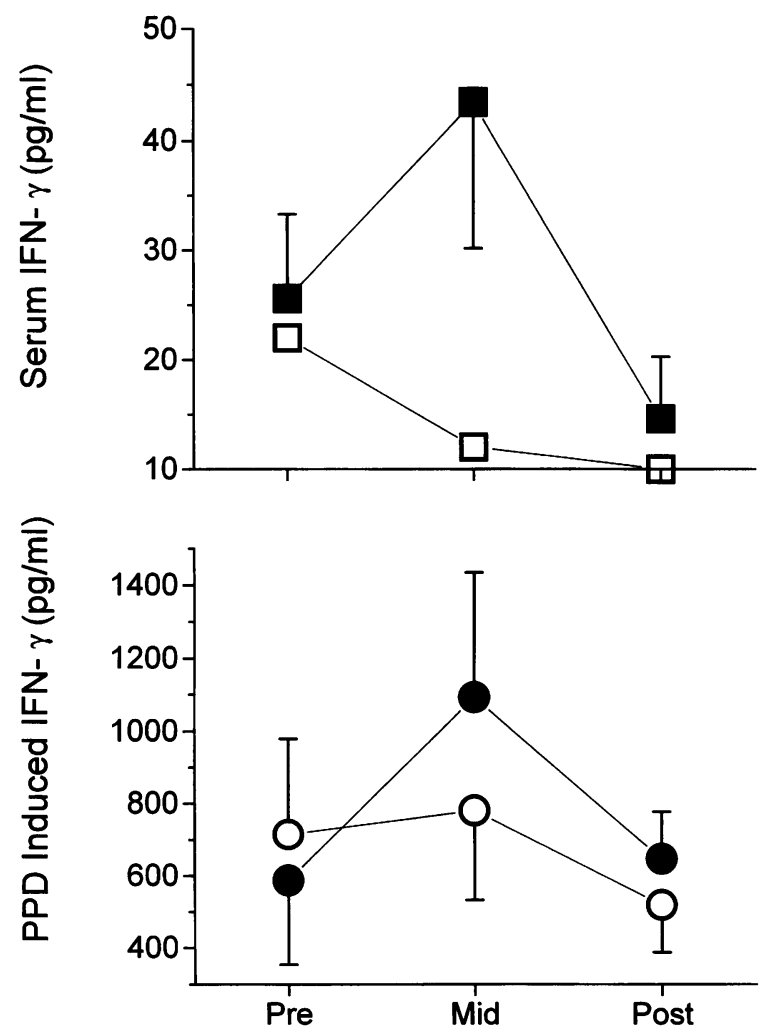

Thalidomide/Placebo Treatment

FIG. 1. The effect of thalidomide treatment on IFN $\boldsymbol{\gamma}$ production

IFN $\gamma$ levels were evaluated by ELISA in the serum of patients (top) and in the supernatants of PBMCs obtained from patients and stimulated in vitro with PPD (bottom). (Top) 14 patients were treated for 14 days with $300 \mathrm{mg} /$ day of thalidomide $(\boldsymbol{\square})$. Serum levels were significantly higher after 7 days on the drug (Mid) relative to the pretreatment time point $(p=0.05)$ and significantly lower after discontinuation of treatment (Post) $(p=0.005)$. Ten patients were treated with MDT and placebo $(\square)$. (Bottom) release of IFN $\gamma$ into the culture supernatants by patient PBMCs stimulated in vitro with PPD before, during, and after thalidomide (O) or placebo treatment (O). Results are means \pm one SEM.

lease was transient, and, for most patients, levels of cytokine released by the cells returned to baseline or higher after thalidomide treatment was discontinued (Fig. 2 and Table 5). It is noteworthy that cells from patients receiving placebo failed to show any reduction in TNF $\alpha$ release at the midstudy time point, regardless of their HIV-1 status (Fig. 2 and Table 5).

Since the effect of thalidomide treatment on TNF $\alpha$ release by the cells in response to LPS was most pronounced at the midpoint of treatment

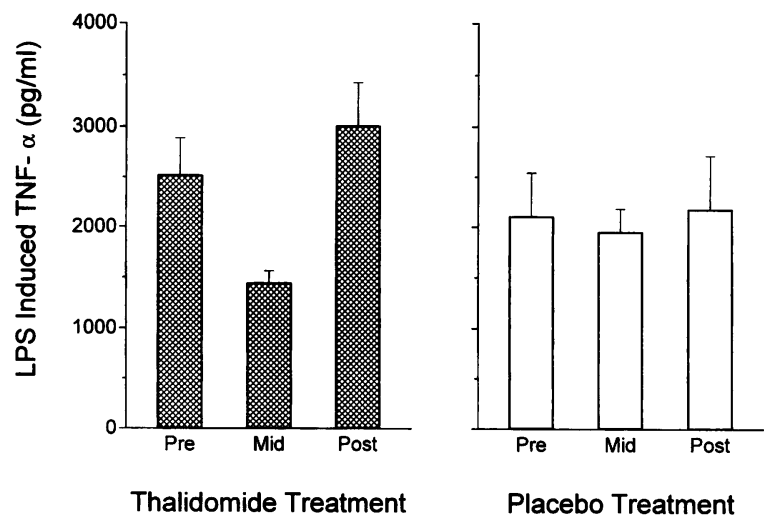

FIG. 2. The effect of thalidomide treatment on TNF $\alpha$ production in vitro

TNF $\alpha$ levels were evaluated by ELISA in the supernatants of PBMCs obtained from $12 \mathrm{HIV}^{-} 1^{-}$and 10 HIV $-1^{+}$patients treated with MDT and $300 \mathrm{mg} /$ day of thalidomide (left) and eight patients (5 $\mathrm{HIV}-\mathrm{l}^{-}$) treated with MDT and placebo (right). PBMCs obtained before (Pre) on Day 7 of treatment (Mid) and 5 days after (Post) the first cycle of treatment were stimulated with LPS and culture supernatants were collected for assay. Results are means \pm one SEM for samples obtained from all patients tested. TNF $\alpha$ levels were significantly lower during treatment with thalidomide relative to levels before $(p=0.001)$ or after $(p=0.002)$ thalidomide.

and waned when thalidomide treatment ended, a group of patients from Group II was treated with a second 14-day cycle of thalidomide after a 7-day washout period. When PBMCs were obtained from patients during the second cycle of thalidomide treatment and stimulated with LPS, the effect on TNF $\alpha$ levels was reproduced (Fig. 3).

To test whether the thalidomide-induced reduction in $\mathrm{TNF} \boldsymbol{\alpha}$-producing capacity of PBMC was correlated with lower circulating TNF $\alpha$ levels in the patients, serum TNF $\alpha$ levels were measured during two consecutive 14-day cycles of thalidomide treatment (with washout periods between treatment cycles). When serum from patients was assayed for TNF $\alpha$ during repeated cycles of thalidomide treatment, a decrease in serum TNF $\alpha$ was observed at the midpoint of each thalidomide treatment (Fig. 3). In addition, mRNA was isolated from unstimulated PBMCs of patients receiving the sequential cycles of thalidomide treatment. Analysis of levels from unstimulated PBMC during thalidomide treatment, showed decreases in the number of copies of TNF $\alpha$ mRNA, mirroring the decrease in serum TNF $\alpha$ levels (Fig. 3). 
TABLE 5. BCG induced cytokine production by PBMC

\begin{tabular}{|c|c|c|c|c|c|c|}
\hline \multirow[b]{2}{*}{ Cytokine } & \multicolumn{3}{|c|}{ Thalidomide treatment $(n=18)^{a}$} & \multicolumn{3}{|c|}{ Placebo treatment $(n=8)^{a}$} \\
\hline & Pre & Mid & Post & Pre & Mid & Post \\
\hline TNF $\alpha$ & $1871 \pm 601$ & $1274 \pm 565$ & $2504 \pm 793$ & $1875 \pm 797$ & $2283 \pm 775$ & $1815 \pm 790$ \\
\hline IFN $\gamma$ & $218 \pm 122$ & $549 \pm 330$ & $218 \pm 58$ & $197 \pm 69$ & $236 \pm 79$ & $231 \pm 55$ \\
\hline
\end{tabular}

TNF $\alpha$ and IFN $\gamma$ levels were assayed by ELISA (as described in Materials and Methods) in the supernatants of PBMCs obtained from patients treated with either thalidomide or placebo. Results are expressed as $\mathrm{pg} / \mathrm{ml}$.

${ }^{a}$ The means for the number of patients shown in the parentheses \pm SEM are given.

IL-1 PRODUCTION. TNF $\alpha$ and IL-1 are usually produced by PBMC in response to the same stimuli in vitro (e.g., LPS). Since thalidomide treatment of tuberculosis patients reduces the capacity of
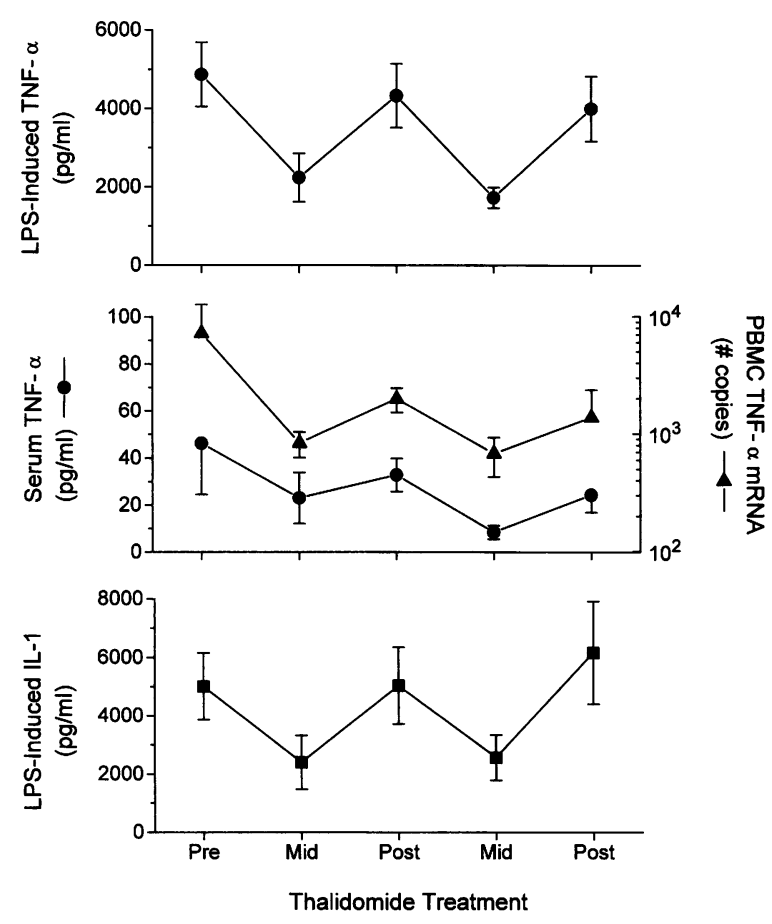

FIG. 3. The effect of thalidomide treatment on TNF $\alpha$ and IL-1 production

In vitro LPS induced release of TNF $\alpha$ (top) and IL-1 (bottom) from PBMC. Serum TNF $\alpha$ levels and PBMC TNF $\alpha$ mRNA levels (middle). Results are means \pm one SEM for samples obtained from five patients treated with two 14-day cycles of thalidomide (Material and Methods). TNF $\alpha$ mRNA levels and LPS induced TNF $\alpha$ were significantly lower as compared with the pretreatment levels $(p<0.008$ and $p<$ 0.005 respectively).
PBMC to release TNF $\alpha$, it was important to determine whether the production of other inflammatory cytokines, such as IL-1, was similarly affected. We therefore examined the plasma and PBMC for production and release of IL-1. IL-1 was not detected in either plasma or serum from patients receiving thalidomide. However, IL-1 release by LPS-stimulated PBMC in vitro was reduced by thalidomide treatment similar to the reduction in TNF $\alpha$ levels (Fig. 3). IL-1 levels rebounded after cessation of thalidomide treatment, but when thalidomide treatment was resumed, IL-1 levels again were reduced at the treatment midpoint (Fig. 3). No significant effect on IL-1 mRNA levels expressed by freshly tested PBMC was observed in response to multiple cycles of thalidomide treatment (Table 6).

\section{Effect of Thalidomide on Body Weight}

Because thalidomide treatment causes a decrease in serum TNF $\alpha$, and TNF $\alpha$ has been implicated in wasting, the effect of thalidomide on body weight was evaluated daily during the first cycle of treatment. Daily administration of thalidomide resulted in a significant enhancement of

TABLE 6. IL-1 $\beta$ mRNA ${ }^{a}$ in PBMC from patients $^{b}$ during thalidomide treatment

\begin{tabular}{lcccc}
\hline Pre & Mid & Post & Mid & Post \\
\hline $20 \pm 9$ & $14 \pm 5$ & $16 \pm 6$ & $16 \pm 11$ & $13 \pm 7$
\end{tabular}

${ }^{m}$ RNA copy number $\times 10^{-3}$ per 10 ng total RNA.

${ }^{b} n=5$ patients tested. Results are means \pm SEM. 
weight gain in tuberculosis patients (Fig. 4). Some of the patients receiving placebo also gained weight, but to a lesser extent than the thalidomide treated patients. Both HIV- ${ }^{-}$and HIV- $\mathrm{I}^{+}$patients gained weight similarly (not shown). Upon withdrawal of thalidomide at 14 days, a reversal or cessation of weight gain was observed (Fig. 4).

In order to evaluate the reproducibility and persistance of the effect of thalidomide on weight gain, six of the Group II (New York only) patients were treated with sequential cycles of thalidomide or placebo in the following manner. Three patients were treated with a 14-day cycle of thalidomide, followed by a 7-day washout period, and then retreatment with thalidomide (Fig. 5). Three patients were treated with a cycle of thalidomide, followed by a washout period, followed by treatment with placebo (Fig. 5). Finally, two patients were treated with placebo during the first 14-day cycle, followed by a washout period, and then followed by thalidomide treatment (Fig. 5). The rate of weight gain was significantly higher while patients were receiving thalidomide, whether or not they had been treated with thalidomide in previous cycles. Moreover, the rate of weight gain was much lower while patients were on placebo or during the washout period. If patients were treated in the first cycle with thalidomide and then received placebo during the second treatment cycle, they stopped gaining weight at the accelerated rate, but these patients did not lose the weight gained during the thalidomide treatment. As a group, patients on placebo gained less weight than patients on thalidomide. This occurred despite maintaining a relatively constant caloric intake before, during, and after thalidomide and/or placebo treatment (Fig. 6). Thus, the weight gain during thalidomide treatment did not appear to be dependent on a change in caloric intake.

\section{DISCUSSION}

TNF $\alpha$ is an important regulatory cytokine of host response to bacterial and parasitic infections (1). A delicate balance must be maintained: enough TNF $\alpha$ must be produced to stimulate host immunity, but overproduction of this cytokine may have extremely deleterious effects. Increasing attention has therefore focussed on thalidomide, a therapeutic agent that appears to down-regulate TNF $\alpha$ overproduction without completely suppressing it (12-14). To determine whether tha- lidomide might affect TNF $\alpha$ production and the subsequent disease course in tuberculosis, we carried out a two-part placebo-controlled pilot study of thalidomide treatment in patients with tuberculosis and wasting. Our observations showed that thalidomide at a dose of $300 \mathrm{mg}$ per day for 14 days was well tolerated with minimal side effects. No adverse clinical manifestations were noted in either patients infected with $M$. tuberculosis alone or patients with dual infections of tuberculosis and HIV-1.

For thalidomide to be clinically useful in controlling the consequences of excessive TNF $\alpha$ production during a chronic infectious process, it should not adversely affect other parameters of host immunity. Therefore, the possible effects of thalidomide treatment on host immunity in tuberculosis were investigated in a number of ways. First, delayed type hypersensitivity was assessed in each patient during the study. Our results show that the response to PPD was not diminished during or after administration of thalidomide, as quantitated by skin test responsiveness (Table 2). On the contrary, there was evidence of enhanced reactivity. That is, $\mathrm{HIV}_{-1}{ }^{-}$ and HIV $-1^{+}$patients who were initially nonresponsive to mycobacterial antigen, developed skin test responsiveness during the administration of thalidomide. This was not observed in placebo-treated patients, suggesting that the observed reactivity was not due to a booster effect of repeated PPD administration.

Administration of thalidomide did not cause any significant changes in total leukocyte count or differential cell count (Table 3), nor was there any significant effect on lymphocyte subset distribution in either $\mathrm{HIV}-\mathrm{I}^{+}$or HIV-1- ${ }^{-}$patients. In addition, when PBMCs were isolated from patients during the course of thalidomide treatment, there was no change in the proliferative capacity of the PBMCs following in vitro stimulation with antigens (Table 4). Thalidomide did not suppress cell-mediated immunological responses in vitro during the treatment cycle.

The effect of thalidomide treatment on cytokine production was evaluated in all patients during the study. Thalidomide treatment resulted in increased levels of IFN $\gamma$ secretion both in vivo, in the serum of patients, and in vitro by PBMCs stimulated with mycobacterial preparations (Fig. 1). This effect was observed in both HIV $-1^{+}$and HIV- $1^{-}$patients. The apparent immunostimulatory effect of thalidomide was limited to the treatment cycle, and when thalidomide therapy ceased, the effect waned (Fig. 1). 




FIG. 4. The effect of thalidomide treatment on weight gain

Weight gain is expressed as the mean of the percent of body weight gained relative to starting weight \pm one SEM for 21 patients treated for 14 days with MDT and $300 \mathrm{mg} /$ day of thalidomide (O) and eight patients treated for 14 days with MDT and placebo $(\boldsymbol{\Delta})$. The arrow above the figure denotes the duration of treatment with thalidomide or placebo. On Days 15 and 21 , weight gain in all thalidomide-treated patients was significantly higher than that of placebo-treated patients $(p=0.003$ and 0.04 , respectively).

The effect of thalidomide treatment on TNF $\alpha$ production, both in vitro and in vivo, was pronounced. PBMCs were isolated from patients during the study and then stimulated in vitro, with a variety of agonists. Thalidomide treatment of tuberculosis patients was clearly associated with a reduced production of TNF $\alpha$ in vitro by stimulated PBMCs (Fig. 2). The effect of thalidomide treatment on TNF $\alpha$ levels was transitory, and cytokine levels returned to baseline when thalidomide treatment ended. However, when patients were treated with a second 14-day cycle of thalidomide, following a 7-day washout period, the effect on TNF $\alpha$ was repeated and TNF $\alpha$ levels in vitro again declined (Fig. 3). These results showed that continuous thalidomide treatment was necessary to maintain the reduction in PBMC capacity to produce TNF $\alpha$.

Simultaneously, we investigated the effect of thalidomide treatment on TNF $\alpha$ production in the patients. It had previously been noted that circulating TNF $\alpha$ levels are notoriously difficult to detect (16). In our study, serum or plasma TNF $\alpha$ was not detected by the Endogen ELISA kit

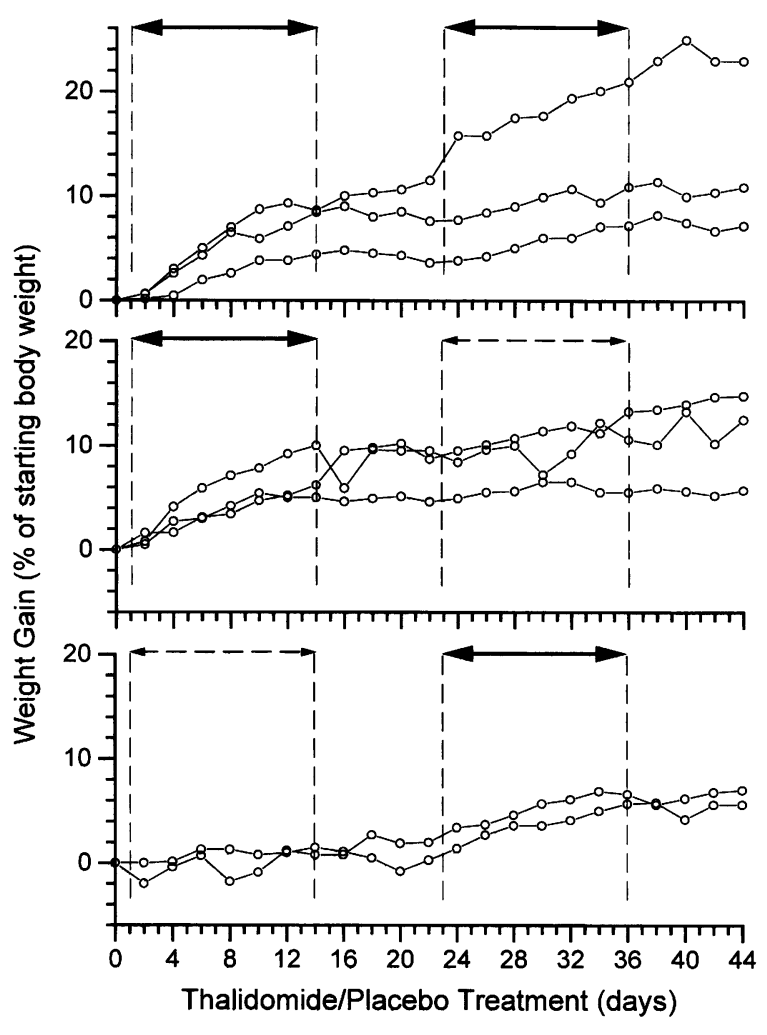

FIG. 5. The effect of thalidomide on weight gain during two cycles of treatment

Weight gain was expressed as the percent of body weight gained relative to starting weight for each patient tested. Patients were evaluated during two cycles of treatment with thalidomide followed by a washout period and another cycle of thalidomide (three patients) (top), thalidomide followed by a washout and a placebo cycle (three patients) (middle), and placebo followed by a washout and a thalidomide cycle (two patients) (bottom). The arrows above the graphs denote thalidomide treatment (solid) and placebo treatment (broken). Statistical analyses of the differences in the slopes of each curve showed that weight gain was significantly different on thalidomide versus washout or placebo $(p=0.0043$ and 0.031 , respectively) and not significantly different between placebo and the washout period $(p=0.36)$.

(see Materials and Methods) which detects free, non-receptor-bound TNF $\alpha$. However, TNF $\alpha$ was detected by the Medgenix kit (Materials and Methods) which detects total TNF $\alpha$, whether or not it is bound to its receptor. When serum from patients was assayed with the latter kit, a reduction in levels of TNF $\alpha$ in the circulation of patients treated with thalidomide was noted. Again, the reduction was dependent on continuous treatment with thalidomide; when thalidomide was not given, TNF $\alpha$ levels returned to 


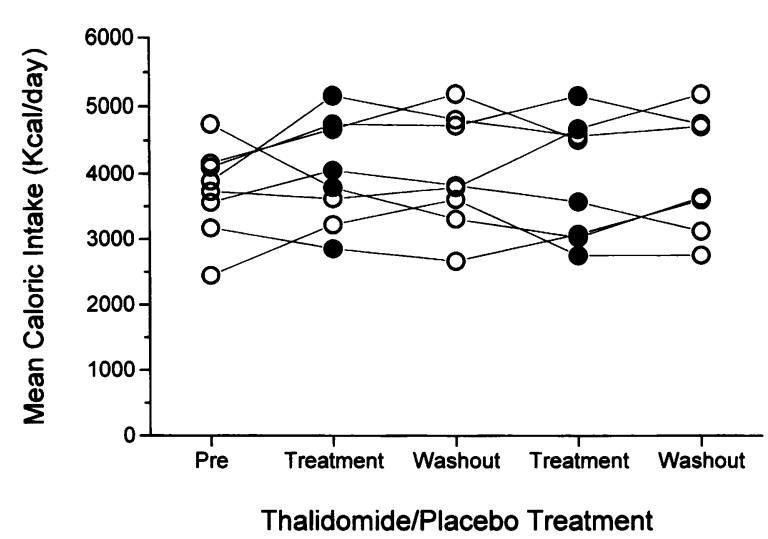

FIG. 6. Effect of thalidomide treatment on caloric intake in tuberculosis patients

Eight patients were treated as described in Fig. 5 . Results are expressed as mean $\mathrm{kcal} /$ day for each patient. (O) mean caloric intake while on placebo or during the washout period. (O) mean caloric intake while on thalidomide.

baseline. TNF $\alpha$ mRNA levels in unstimulated circulating leukocytes, a sensitive assay for TNF $\alpha$ gene activation, were also decreased during thalidomide treatment (Fig. 3). This is a possible explanation for why cells from these patients release less of this cytokine in vitro in response to agonists.

We had previously shown that a short thalidomide pulse in vitro to PBMCs stimulated with LPS inhibited TNF $\alpha$ without affecting levels of IL-1 production (13). In the present study, the relatively long cycle of thalidomide administration in vivo had no significant effect on serum IL-1 or IL-1 mRNA levels. However, thalidomide treatment in vivo did cause a reduction in IL-1 release by LPS stimulated PBMCs in vitro (Fig. 3 ). These apparently contradictory effects may be due to the sensitivity of PBMC IL-1 production to TNF $\alpha$ levels, which are in turn, modulated by the duration of thalidomide treatment in vivo.

All patients in this study gained weight during treatment with thalidomide. While some of the patients treated with MDT and placebo also gained weight, the weight gain of patients treated with thalidomide was significantly greater than that of the placebo-treated group. After the cessation of thalidomide treatment, some patients lost some weight while other ceased to gain weight (Figs. 4 and 5). These observations suggest that thalidomide could be used clinically to accelerate weight gain during anti-tuberculosis therapy. In this study, weight gain was not a consequence of increased caloric intake, since there was no significant difference in this parameter between thalidomide and placebo treatment. Some of the thalidomide in- duced weight gain persisted for at least 4 weeks after the end of thalidomide treatment (Fig. 5), suggesting that the weight gain was not due merely to transient fluid retention, nor was it due solely to MDT, since placebo-treated patients did not gain as much weight. However, measurement of total body and/or lean body mass are required to conclusively demonstrate the nature of the weight gain during thalidomide treatment. The results presented here suggest that thalidomide may exert a metabolic effect, perhaps mediated through TNF $\alpha$ levels. The nature of this potential metabolic change is being investigated in ongoing studies.

The results of our present study indicate strongly that thalidomide reduces TNF $\alpha$ production in both uncomplicated tuberculosis and tuberculosis associated with HIV-1 infection. The decrease in TNF $\alpha$ production was not associated with any clinical deterioration or negative effects on host immunity. How treatment with thalidomide impacts on the cytokine regulatory circuit and results in reduced TNF $\alpha$ production is as yet unknown. Our results suggest that cachexia may, at least in part, be mediated by chronic production of TNF $\alpha$. Thalidomide therapy might thus be instrumental in reversing the debilitating symptoms of $M$. tuberculosis infection as well as in another syndrome with associated cachexia, the acquired immunodeficiency syndrome (17). Also, the reduction in TNF $\alpha$ induced by thalidomide may have profound effects on other physiologic pathways that are modulated by cytokine levels. For example, we have recently shown that thalidomide induced reduction in TNF $\alpha$ is associated with a reduction in activation of latent HIV-1 in vitro (18). The mechanism of this effect and its potential effect on clinical course is currently under investigation in our laboratory.

\section{ACKNOWLEDGMENTS}

We thank the nurses Sumalee Ammarinsangpen, Jinda Tongsuradet and Amporn Suwanprasert, from the Chiang Mai tuberculosis control center, for making this study possible. Dr. Attapon Cheepsatayakorn is acknowledged for help with the radiology. We also thank Cynthia Seidman, Dacia Vasquez, and Jolanta Diakun from the Dietary Service at the Rockefeller University GCRC, and the nurses and staff of the Rockefeller University Hospital; Marguerite Nulty for typing the manuscript; Judy Adams for help with the figures; and Jason Tian for help with the statistical analysis. Dr. Victoria Schauf helped initiate 
these studies. Dr. Victoria Freedman's help in writing the manuscript is gratefully acknowledged. This study was supported in part by U.S. Public Health Service Grants AI 24775, AI 33124 , and AI 07012, a Rockefeller University General Clinical Research Center Grant (MO1-RR00102), Grunenthal GMBH, and Celgene Corporation.

\section{REFERENCES}

1. Beutler B, Cerami A. (1988) The biology of cachectin-TNF: A primary mediator of the host response. Annu. Rev. Immunol. 7: 625-655.

2. Barnes PF, Fong S-J, Brennan PF, Twomey PE, Mazumder A, Modlin RL. (1990) Local production of tumor necrosis factor and IFNgamma in tuberculous pleuritis. J. Immunol. 145: 149-154.

3. Takashima T, Ueta C, Tsuyuguchi I, Kishimoto S. (1990) Production of tumor necrosis factor alpha by monocytes from patients with pulmonary tuberculosis. Infect. Immun. 58: 3286-3292.

4. Ogawa T, Uchida $\mathrm{H}$, Kusumoto $\mathrm{Y}$, Mori $\mathrm{Y}$, Yamamura Y, Hamada S. (1991) Increase in tumor necrosis factor alpha- and interleukin-6-secreting cells in peripheral blood mononuclear cells from subjects infected with Mycobacterium tuberculosis. Infect. Immun. 59: 3021-3025.

5. Valone SE, Rich EA, Wallis RS, Ellner JJ. (1988) Expression of tumor necrosis factor in vitro by human mononuclear phagocytes stimulated with whole Mycobacterium bovis BCG and mycobacterial antigens. Infect. Immun. 56: 3313-3315.

6. Kindler V, Sappino A-P, Grau GE, Piguet P-F, Vassalli $P$. (1989) The inducing role of tumor necrosis factor in the development of bactericidal granulomas during BCG infection. Cell 56: 731-740.

7. Havell EA. (1989) Evidence that tumor necrosis factor has an important role in antibacterial resistance. J. Immunol. 143: 2894-2899.

8. North RJ, Izzo A. (1993) Granuloma formation in severe combined immunodeficient (SCID) mice in response to progressive BCG infection. Am. J. Pathol. 142: 1959-1966.

9. Tracey KJ, Wei H, Manogue KR, et al. (1989)
Cachectin/tumor necrosis factor induces cachexia anemia and inflammation. J. Exp. Med. 167: 1211-1227.

10. Cotran RS, Kumar V, Robbins SL (eds). (1989) Pathologic Basis of Disease. 4th Ed. W. B. Saunders, Philadelphia.

11. van der Poll T, Jansen J, van Leenen $D$, et al. (1993) Release of soluble receptors for tumor necrosis factor in clinical sepsis and experimental endotoxemia. J. Infect. Dis. 168: 955-960.

12. Sampaio EP, Kaplan G, Miranda A, et al. (1993) The influence of thalidomide on the clinical and immunologic manifestation of erythema nodosum leprosum. J. Infect. Dis. 168: $408-414$.

13. Sampaio EP, Sarno EN, Gallily R, Cohn ZA, Kaplan G. (1991) Thalidomide selectively inhibits TNF $\alpha$ production by stimulated human monocytes. J. Exp. Med. 173: 699-703.

14. Sarno EN, Grau GE, Vieira LMM, Nery JA. (1991) Serum levels of tumour necrosis factor-alpha and interleukin-1 beta during leprosy reactional states. Clin. Exp. Immunol. 84: 103-108.

15. Moreira AL, Sampaio EP, Zmuidzinas A, Frindt P, Smith KA, Kaplan G. (1993) Thalidomide exerts its inhibitory action on tumor necrosis factor- $\alpha$ by enhancing mRNA degradation. J. Exp. Med. 177: 1675-1680.

16. Aukrust P, Liabakk N-B, Muller F, Lien E, Espevik T, Froland SS. (1994) Serum levels of tumor necrosis factor- $\alpha$ (TNF $\alpha$ ) and soluble TNF receptors in human immunodeficiency virus type 1 infection- correlation to clinical, immunologic, and virologic parameters. J. Infect. Dis. 169: 420-424.

17. Johnson BJ, McMurray D. (1994) Cytokine gene expression by cultures of human lymphocytes with autologous Mycobacterium tuberculosis-infected monocytes. Infect. Immun. 62: 1444-1450.

18. Makonkawkeyoon S, Limson-Pobre RNR, Moreira AL, Schauf V, Kaplan G. (1993) Thalidomide inhibits the replication of human immunodeficiency virus type 1. Proc. Natl. Acad. Sci. U.S.A. 90: 5974-5978. 